\title{
LEISHMANIA, HERPETOMONAS, AND CRITHIDIA IN FLEAS.
}

\author{
By Dr CARLO BASILE, D.T.M., R.C.PH.S. (LoNdon), \\ Libero docente (University of Rome). \\ (With Plates XXI and XXII.) \\ INTRODUCTION.
}

Most of the observations recorded in this paper would have been published in 1915, if my duties as a doctor and as an officer had not called me to the Army.

Following on my experimental researches (1910-1915), on the transmission of visceral leishmaniasis by means of fleas (Ctenocephalus canis and Pulex irritans), a very interesting problem arose which still rivets the attention of students of this subject.

In the intestine of various insects and of fleas in particular, certain Protozoa of the herpetomonad and crithidial types have been observed. The morphological, biological and experimental study of these Protozoa in relation to Leishmania is particularly interesting.

Patton maintained that this parasite is a true Herpetomonas, and called "herpetomoniasis" those infections which we now call "leishmaniasis." Minchin and Wenyon, on the other hand, observed that although Leishmania in some stages presents morphological characteristics almost identical with Herpetomonas it is distinguished by the fact that Herpetomonas is only found in insects, in whose intestines the different species undergo their complete developmental cycle (flagellate and non-flagellate), while Leishmania completes its cycle in two hosts, one of which is a vertebrate and the other an invertebrate. In the vertebrate host Leishmania produces a disease with characteristic symptoms- "Leishmaniasis."

\section{BIOLOGY AND MORPHOLOGY OF LEISH MANIA.}

The biological and morphological characteristics of the parasite must be studied, $(a)$ in the vertebrate host; $(b)$ in artificial cultures; $(c)$ in the transmitting host.

(a) Leishmania in the vertebrate host is non-flagellate, it appears either oval (Plate XXI, fig. 1), or round, with a diameter varying from 2 to $4 \mu$; it may also be pear-shaped (Plate XXI, fig. 3) when it may attain a length of $7 \mu$. 
In the parasite we can distinguish two masses of chromatin, the larger is called the nucleus, or trophonucleus, the smaller is called the blepharoplast, or kinetonucleus. Novy has also described a small well defined organ, the rhizoblast. The plasma appears finely granulated and in the large forms shows vacuoles.

The nucleus is rounded and often appears as a compact mass (Plate XXI, fig. 1); occasionally it seems to consist of small grains of chromatin; at times it shows a minute body more deeply coloured, placed centrally or eccentrically, which recalls the karyosome (in sensu lato); very rarely the nucleus is absent.

The blepharoplast is rod-shaped (Plate XXI, fig. 1) or pointed (Plate XXI, fig. 2), almost always detached from, but at times attached to the nucleus (Plate XXI, fig. 2). Visentini, who has carefully studied the morphology of Leishmania in the vertebrate host, thinks that this attachment of the blepharoplast to the nucleus indicates the nuclear origin of the blepharoplast; sometimes the blepharoplast may be absent (Plate XXI, figs. 4, 5).

In parasites derived from haematopoietic organs, the rhizoblast occurs as a minute body or rod placed between the kinetonucleus and the peripheral zone of the parasite. In canine leishmaniasis, smears from such organs reveal the presence of granular forms which I believe to be connected with Leishmania, but I cannot say definitely whether they represent a phase of the developmental cycle and are comparable with the granular forms of trypanosomes, or whether they represent degenerative forms of the parasite.

Reproduction. According to all authorities, Leishmania reproduces itself by simple longitudinal division; division of the nucleus and of the blepharoplast is first observed (Plate XXI, figs. 5, 6); then the plasma divides. This form of agamic reproduction leads to the formation of characteristic bodies consisting of several parasites enclosed in a common protoplasm; Ross suspected a sporulation phase; Nicolle on the other hand, considered them to be parasites enclosed in the plasma of the leucocytes whose nucleus might have become detached in the process of preparing the smear.

Besides the reproduction by simple longitudinal division, from which two individuals morphologically indistinguishable are produced (equal division), I have found that sometimes the longitudinal division gives origin to two individuals which can be morphologically distinguished (unequal division); I call the equal division "homomorphous" and the unequal division "heteromorphous."

In heteromorphous division the blepharoplast takes no part whatever in the process of division, which occurs only in the nucleus (Plate XXI, fig. 7).

Visentini described, without giving any explanation, the existence of parasites consisting of plasma enclosing a nucleus but no blepharoplast (Plate XXI, fig. 4). I have decided that such forms result from an unequal division wherein no division of the blepharoplast occurs, the nucleus dividing into two equal similar nuclei, the plasma also dividing immediately. Two parasites are thus 
produced, one of which presents a nucleus in which the karyosome is not visible, but which contains the maternal blepharoplast, while the other parasite contains no blepharoplast but shows a nucleus with a karyosome (Plate XXI, fig. 7). This explains the appearance already minutely described by Visentini, and leads us to believe in the nuclear origin of the blepharoplast.

The heteromorphous division above described is probably of significance in the life cycle of Leishmania. The development of a parasite which at first is apparently without a blepharoplast is an important phenomenon; I think that some forms of the parasite may represent resistant forms which are of use in the maintenance of the species and which are produced under special conditions. I base this deduction on the fact that I have most frequently met with these forms in the haematopoietic organs of dogs naturally infected with leishmaniasis which was running a chronic course.

(b) Leishmania in artificial cultures. Rogers, who was the first to cultivate Leishmania donovani did so by adding citric acid or citrate of soda in the proportion of 10 per cent. to the spleen or liver juice of individuals affected with leishmaniasis (Indian virus); his experiments being confirmed by Leishman and Statham whilst Longo subsequently cultivated the Mediterranean Leishmania. Nicolle, in 1907, cultivated the parasite in the Novy-McNeal medium, and, in 1908, in the Novy-McNeal-Nicolle medium.

Row, in 1912, obtained the cultural development of Leishmania donovani (Indian virus) in a haemoglobinised saline solution and Visentini used this medium for cultivating Leishmania infantum. Leishmania also develops in Bordet and Gengou's medium, in human blood agar and in Jemma's asciticagar medium. The temperature is of the highest importance in the cultural development of these parasites; they develop at a temperature of between $18^{\circ} \mathrm{C}$. and $25^{\circ} \mathrm{C}$, with the best results at $20^{\circ} \mathrm{C}$. to $22^{\circ} \mathrm{C}$.

After inoculation, from four to five days, on an average, are required before flagellate forms of Leishmania appear in the culture fluid. This development may however be completed in a shorter period. Visentini and I once saw a few flagellates appear 48 hours after cultures on N.N.N. medium had been inoculated with the splenic juice of a dog intensely infected with leishmaniasis. After several days non-flagellated forms reappeared and we regard these post-flagellate forms as representing resistant stages.

During the first hours after inoculation the young non-flagellate parasites resemble the forms seen in the blood (peripheral and from haemopoietic organs); gradually they become pear-shaped (Plate XXI, fig. 8) and the flagellum develops (Plate XXI, fig. 9). Such flagellate piriform parasites usually measure $8-12 \mu$ in length by $2-3 \mu$ in breadth. Besides the pear-shaped parasites there occur some elongated forms (Plate XXI, figs. 10, 11, 12), with a pointed front end from which the flagellum projects. Leishmania in cultures reproduces itself by simple longitudinal division and this leads to the formation of slender elongated parasites (Plate XXI, figs. 13, 14, 15).

After some days, there appear flagellate parasites of large size which may 
measure up to $26 \mu \times 5 \mu$. The appearance of these large forms is evidently due to the artificial conditions prevailing in the culture, and, since many of them show vacuolated plasma, they may well represent degenerative forms.

In addition, elongated and spherical forms of rather large size and with granules scattered in the plasma are to be noted. These forms have been regarded as sexual forms; or (Rogers, Marzinowski) as conjugation forms. I have made repeated and prolonged observations in this regard, using a thermostat at $22^{\circ} \mathrm{C}$, but I have never observed conjugation to take place.

The morphological study of cultural Leishmania both of infantile and canine origin has been carefully followed by Visentini at the Lister Institute. I shall refer to the principal deductions of this writer, dealing chiefly with the morphological identity between the evolutive forms of Leishmania in fleas and the forms encountered in cultures. .

(c) Leishmania in the transmitting host (Ctenocephalus canis, Pulex irritans). Patton, describing the life cycle of the flagellate Protozoa of insects (Herpetomonas and Crithidia), has distinguished three stages--the pre-flagellate, the flagellate, and the post-flagellate. These stages are also to be noted in the cycle of Leishmania in fleas. The pre-flagellate stage is found in the flea's midgut; the flagellate stage in the hind-gut; and the post-flagellate stage in the rectum and faeces; these three stages correspond morphologically to those of Leishmania in cultures.

In the flea's mid-gut, the parasites show the same characteristics which they present in the peripheral blood and in the haematopoietic organs of infected vertebrates; when fixed and coloured they appear rounded, oval, or pear-shaped (Plate XXI, figs. 16-19), they measure on an average $2-4 \mu$ in diameter. The protoplasm assumes a delicate pale blue tint; the nucleus of a reddish violet colour is almost always compact, sometimes granular; the blepharoplast is sometimes absent, as has been noted in parasites from the haematopoietic organs; when it is present it may be rounded or rod-shaped and of the same, or a deeper tint than the nucleus.

Leishmania in this pre-flagellate stage divides longitudinally; first the nuclei and then the plasma. In this manner forms are produced indistinguishable morphologically from those in the haematopoietic organs (Plate XXI, figs. 20, 21, 22).

In some stained preparations however, I have seen certain parasites (Plate XXI, fig. 23), which show two nuclei and a blepharoplast. I have carried out numerous experiments to ascertain whether these might represent sexual forms, but with no success.

In fleas experimentally infected and kept at a temperature of $22^{\circ} \mathrm{C}$., after 36 hours from infection I have observed flagellated forms in the intestine, these are morphologically identical with the pear-shaped or elongated cultural forms which have been already described.

In fact, these flagellate, pear-shaped parasites in fleas have almost the same measurements and appearance as the cultural forms, being $7-9 \mu$ in 
length, exclusive of the flagellum, and $2-4 \mu$ in breadth (Plate XXI, figs. 24, $25,26)$. The blepharoplast is situated in front of the nucleus, to which it is more or less contiguous (Plate XXI, figs. 24, 26); in parasites which are nearing the post-flagellate stage it is situated laterally to the nucleus (Plate XXI, fig. 25). The flagellum may attain a length of $20-22 \mu$; it may develop from a basal granule (Plate XXI, figs. 25, 26, 27), or directly from the blepharoplast (Plate XXI, figs. 28, 29).

The elongated parasites (Plate XXI, figs. 27, 28, 29) are also morphologically identical with the cultural forms; they reach a size of $9-11 \mu$ in length by about $2 \mu$ in breadth; their anterior extremity is pointed; the posterior end is conical, as in the pear-shaped forms. Thus, as in the cultural forms, the various "organellae" may have different shapes and positions in different flagellate individuals.

Leishmania in the flagellate stage reproduces itself in fleas by longitudinal division (Plate XXI, fig. 28); this takes place in the same way as that described for the cultural forms. Either the flagellum, or the blepharoplast, or the nucleus may divide first; the plasma divides last. Two equal parasites are thus produced, and I have never met with unequal division.

In my numerous researches with naturally infected fleas I have very rarely found this flagellate stage; it has seemed to me that this stage may have some connection with the nutrition of the flea; I have more easily found it in fleas whose mid-gut still contained some undigested blood. This observation is of considerable value; if it should be confirmed it will provide a distinctive character between the developmental forms of Leishmania in fleas and the common Herpetomonas and Crithidia of insects, because these latter constantly show all stages, both flagellate and non-flagellate.

The flagellated Leishmania, after more or less active multiplication in the intestine of the flea, undergoes certain modifications in structure; the blepharoplast first approaches the nucleus, in some cases coming to lie beside it, the basal granule follows and, in this stage, the flagellum is attached along the anterior third of the body (Plate XXI, fig. 25). In successive stages the anterior, rounded extremity of the parasite becomes pointed and a very short flagellum projects from it; the posterior end, which was pointed, almost conical, becomes rounded and the parasite assumes a pear-shaped form (Plate XXI, fig. 30); later, the flagellum entirely disappears (Plate XXI, figs. 31, 32), the plasma appears of a red colour, the nucleus, the blepharoplast and the basal granule of a deep red colour; still later, the basal granule disappears, the blepharoplast becomes absorbed in the nucleus (Plate XXI, fig. 33); finally, the nuclear chromatin breaks up into numerous granules distributed in the plasma (post-flagellate stage) (Plate XXI, fig. 34). These post-flagellate bodies become provided with a cystic membrane and then the internal contents stain with difficulty. Cystic forms of flagellate Protozoa have been described; e.g., cysts of Trypanosoma grayi in the mid-gut of Glossina palpalis, which were discovered and described by Minchin. During encystment I have never ob- 
served nuclear multiplication, such as has been described by Wenyon in similar forms of Herpetomona's muscae-domesticae.

The post-flagellate forms of Leishmania, which are expelled with the faeces, help to keep the species in existence and to spread it; this dispersal takes place through the infection per os of other fleas and perhaps also of other insects. My experiments, as also those of Archibald (1914), lead me to believe that these post-flagellate forms are also capable of infecting, by the alimentary tract, dogs and healthy children; this is an example of the contaminative mode of infection.

The description of the developmental cycle of Leishmania in my previous notès has been confirmed by Alvarez and Sergent.

Alvarez described the occurrence of Leishmania, in the pre-flagellate, flagellate and post-flagellate stages, in the intestine and faeces of fleas from a dog infected experimentally with infantile leishmaniasis; Sergent, in collaboration with Lhéritier and Lemaire, described Leishmania from the intestinal contents and faeces of fleas taken from a dog infected naturally with leishmaniasis. The studies of Sergent and his collaborators are highly important, because these writers, repeating my experiments with infected fleas, also succeeded in transmitting leishmaniasis to a healthy dog.

- The study of the developmental cycle of Leishmania in the flea indicates the possibility that there may be two methods of infection of the vertebrate host; the method of inoculation, by the penetration of the parasite through the skin, and the method of contamination, by the post-flagellate forms per os.

This possibility of a double mode of infection explains not only the widespread distribution of leishmaniasis in dogs, but also the domestic localisation of the disease, members of the same family becoming infected by fleas, which themselves infect one another by means of the faeces.

\section{LEISHM ANIA, HERPETOMONAS, CRITHIDIA IN FLEAS.}

The morphological study of the Protozoa of herpetomonad and crithidial types discovered in fleas reveals characteristics which differentiate them from the Leishmania which I have discovered in Ctenocephalus canis and Pulex irritans.

These distinctive characters are most evident in the flagellate stage; in this stage the dimensions of Leishmania in fleas, as given above, are very different from those of the various species of Herpetomonas and Crithidia which have been discovered up to the present in the Pulicidae.

The Flagellates observed by Sangiorgi in fleas (Pulex serraticeps) attained a length of $16 \cdot 6 \mu$ with a breadth varying from $3-6 \mu$. The flagellum in these forms is as long as, or longer than the body; Herpetomonas ctenophthalmi described by Mackinnon in Ctenophthalmus agyrtes also attains a length of $22 \mu$, without including the flagellum, and a breadth of $2 \mu$ (Plate XXII, fig. 1); the Herpetomonas discovered by Wenyon in Pulex irritans is about $30 \mu$ long, 
without the flagellum (Plate XXI, figs. 37, 38); Crithidia pulicis discovered by Porter in Pulex irritans may even reach a length of $65 \mu$, including the flagellum (Plate XXII, fig. 12); and Crithidia hystricopsyllae discovered by Mackinnon in Hystricopsylla talpae is $18 \mu$ long, without the undulating membrane and the flagellum (Plate XXII, fig. 8). Forms notably larger than Leishmania are found also in Herpetomonas ctenopsillae described by Laveran and Franchini.

The anterior end of the young pear-shaped forms of Leishmania is rounded and is crossed by the flagellum, while in the Insectan Flagellates it is always pointed and drawn out along the flagellum. The posterior end of these forms in Leishmania is pointed and almost conical, while in the insectan flagellates it is rounded. Leishmania also sometimes shows the anterior end slightly pointed and the posterior end rounded (Plate XXI, fig. 31), but in this case the parasites are always short and thick, with a very short flagellum, and the blepharoplast is very close, or attached to the nucleus; these individuals represent only a stage leading on to the post-flagellate form.

The elongated forms of Leishmania in fleas, which are absolutely identical with the cultural forms, show a pointed anterior end, from which the flagellum projects, and a posterior end, which is abruptly truncated, almost conical. It is to be noted, however, that in the Herpetomonas described by Mackinnon in Ctenophthalmus agyrtes (Plate XXII, figs. 1-4), and by Wenyon in Pulex irritans, the posterior end is drawn out in a filiform prolongation (Plate XXI, figs. 37,38$)$.

Finally, Leishmania is differentiated from Crithidia by the complete absence of an undulating membrane (Plate XXII, figs. 8, 12, 13, 14, 15).

Herpetomonas and Crithidia of fleas, according to Mackinnon, Nöeller and Fantham, may show large forms, which are rounded or elongated (Plate XXII, figs. $5,6,11$ ); in this case the ends are rounded or obtuse, the parasites possess a large nucleus and blepharoplast, and many granules distributed through the vacuolar plasma (Plate XXII, figs. 8, 11); the flagellum in these forms is very short and is continued into the body of the parasite as a conspicuous rhizoblast, more or less deeply coloured. I have never seen similar forms in the developmental cycle of Leishmania in fleas.

In studying the relations between Leptomonas of Ctenocephalus canis and Leishmania of human and canine origin, Chatton has recently observed that in cultures of Leptomonas ctenocephali large forms appear which he calls "aciculées flagellés," which present a body "rubanné" and "torder." We can thus distinguish at once between the cultural forms of Leptomonas ctenocephali and those of Leishmania.

Also the Herpetomonas discovered by Balfour in Pulex cleopatrae has certain stages, the dimensions and morphological characteristics of some stages of Leishmania in human and canine fleas; these, however, are differentiated morphologically in other flagellate stages, when the posterior end is filiform, as in the Herpetomonas found by Mackinnon in Ctenophthalmus agyrtes and by 
Wenyon in Pulex irritans, while in Leishmania, as has been already stated, the posterior end is conical.

It can be deduced from these indications that an accurate study of the complete developmental cycle of the various Herpetomonas and Crithidia already described in fleas reveals certain morphological differences between these and the developmental forms of Leishmania in the same insects; on the other hand, the morphological study, if it be not supplemented by biological and experimental data, may be sometimes insufficient.

In this respect I repeat to-day what I have written ever since I began these studies in 1911, " given the constant presence of Protozoa, often morphologically similar, in the intestine of insects, anyone who studies their developmental cycle rather than their morphology is able, by means of experiments, to infer whether the said Protozoa are etiological agents of disease."

It is, in fact, necessary to bear in mind that the herpetomonad and crithidial forms found in the intestine of fleas are not always Insectan Flagellates, since developmental stages of trypanosomes which possess a herpetomonad or crithidial phase also occur in fleas. Minchin and Thomson found in the rat flea (Ceratophyllus fasciatus) the developmental stages of Trypanosoma lewisi; this parasite, in some of its forms, assumes all the characteristics of Herpetomonas; similar phases of $T$. lewisi have also been discovered in Ctenocephalus serraticeps (Nöeller), in Pulex irritans (Wenyon), and in Xenopsylla cheopis, experimentally infected; we cannot, therefore, exclude the possibility that they may be found in fleas infected in nature.

In reference to the relations between pathogenic forms of Herpetomonas and Trypanosomes the researches dealing with Schizotrypanum cruzi are interesting; in the digestive tract of Triatoma megistus taken from the houses of Minas Geraes, Brazil, Chagas observed Protozoa of the herpetomonad type; monkeys inoculated with the intestinal contents of these Hemiptera became infected with Trypanosomes; this discovery led Chagas to ascertain the existence of a trypanosomiasis previously unknown, which from its clinical symptomatology has been called "parasitic thyroiditis"; the parasite, on account of certain characteristics of its developmental cycle, has been called Schizotrypanum cruzi.

Results identical with those of Chagas have been obtained by Lafont in the island of Mauritius, and by Carini at San Paulo. Recently, also, Tejera when examining the intestinal contents of Rhodnius prolixus in Venezuela found these Hemiptera infected with flagellates which, when inoculated into animals, produced a trypanosome-infection identical with that caused by Schizotrypanum cruzi. These interesting investigations of Chagas into trypanosomiasis, or parasitic thyroiditis, indicate the importance which the researches of experimental protozoology upon the various Herpetomonas and Crithidia found naturally in fleas have in relation to leishmaniasis.

Since 1910 I have been seeking to solve the important problem of the pathogenic significance of the Protozoa of fleas taken from children and dogs 
suffering from visceral leishmaniasis in the Mediterranean region. I have carried out experiments the results of which I will briefly relate. Fleas infected with parasites which I defined as of "leishmanial type," taken from dogs and children infected with leishmaniasis, were put upon healthy dogs. These parasites produced in healthy dogs an infection with the characteristic symptomatology of leishmaniasis; and characteristic leishmanial forms were found in the haematopoietic organs. Similar parasites in fleas were inoculated intraperitoneally into white mice and produced in these animals an infection which has been determined by microscopical examination of the internal organs to be identical with leishmaniasis of infantile or canine origin.

My investigations have been confirmed by Sergent, Lhéritier and Lémaire (1912).

While I, and subsequently Sergent and his collaborators, have made use of fleas taken from children and dogs infected with leishmaniasis, Sangiorgi (1911) began a series of experimental control investigations with a Herpetomonas in Ctenocephalus serraticeps, of unknown origin; unfortunately, the dogs of which he made use died a few days after the beginning of the experiment and before Sangiorgi was able to arrive at any conclusion.

A. Porter for a long period of time allowed fleas (Pulex irritans) infected with parasites of the Herpetomonas-type to suck blood from her arm every day, but she did not note any infection in herself. Nöeller, also, obtained negative results from the intraperitoneal inoculation of dogs with the intestinal contents of Ctenocephalus serraticeps infected with Herpetomonas ctenocephali; Negri obtained similar negative results from puppies (not published), and Chatton from white mice (Mus albinus).

To these negative results we can oppose a series of positive results.

Laveran and Franchini obtained in young rats an infection of the internal organs with parasites of a "leishmanial type," by inoculation, by natural means (puncture of the skin and by the digestive tract), making use of dogfleas naturally infected with Herpetomonas ctenocephali. They obtained similar results also with rat-fleas (Ceratophyllus fasciatus) infected with Herpetomonas pattoni, with Melophagus ovinus infected with Crithidia melophagia and with Anopheles infected with Crithidia fasciculata, with Sarcophaga hemorroidalis, Phlebotomus, and Blatta orientalis infected with protozoa of herpetomonad and crithidial types, and finally by injecting cultures of Herpetomonas ctenocephali and of Crithidia melophagia.

The same writers prepared an emulsion in physiological saline solution of the internal organs of those rats experimentally infected with herpetomoniasis and injected it into dogs and monkeys, in which animals they obtained the reproduction of an infection not distinguishable from that which is observed after inoculation with Leishmania.

Fantham and Porter have experimentally demonstrated that Insectan Flagellates, such as Herpetomonas jaculum (Léger), of Nepa cinerea, Herpetomorias ctenocephali (Fantham), Herpetomonas stratiomyae, Herpetomonas pedi- 
culi, and Crithidia gerridis, if inoculated intraperitoneally, or if ingested, can live and multiply in the blood and in the internal organs of young rats and of dogs and produce in these animals an infection indistinguishable from visceral leishmaniasis.

In later experiments Fantham and Porter have also demonstrated that a similar herpetomoniasis can be produced also in cold-blooded animals; they have, in fact, produced an infection with Herpetomonas jaculum and Crithidia gerridis, both by natural means (the digestive tract) and by experimental means (inoculation) in fishes (Gasterosteus aculeatus), frogs, toads, lizards (Lacerta vivipara) and in snakes (Tropidonotus natrix), parasites being present in the internal organs indistinguishable from those met with in leishmaniasis.

But in addition to these experiments the investigations of natural herpetomoniases in animals are highly interesting.

Dutton and Todd in 1903 observed the natural occurrence of a Herpetomonas in the blood of a rat in Gambia; Fantham and Porter in 1909 again discovered this Herpetomonas in the blood of rats inoculated with spirochaetosis.

Sergent, Lhéritier and Lémaire in prosecuting their researches in Biskra on Tarentola mauritanica established that, in making cultures of the internal organs of Tarentola on blood agar, in $\mathbf{1 5 \cdot 7}$ per cent. of the cases forms of Herpetomonas morphologically indistinguishable from the cultural forms of Leishmania were obtained; on this discovery they based the hypothesis that Tarentola mauritanica may be a "reservoir" host of Leishmania tropica.

Léger has recently affirmed that 2 per cent. of the saurians of the species Anolis show a blood infection of Leptomonas (Leptomonas henrici) which he believes to be of intestinal origin because the same Leptomonas was found in 50 per cent. of the individuals examined.

These facts now known to science show that in mammals, as in fishes, reptiles and amphibia (especially in those which are insectivorous) natural herpetomoniases exist, or an infection with an insectan Herpetomonas may be induced, either by way of the digestive tract or by inoculation. Herpetomoniasis appears to be an infection which until to-day has not been distinguishable, either by the morphological and biological characteristics of the parasite or by its symptoms, from visceral leishmaniasis. .

In my previous work on this subject I have already expressed my belief that the leishmaniases are produced by Protozoa (Herpetomonas and perhaps also Crithidia) which have adapted themselves to live and multiply in vertebrate hosts; this adaptation is easier in the case of the vertebrate on which the insects are ectoparasitic.

The same species of Herpetomonas of insects, under different conditions, may not have any pathogenic action, or may produce pathogenic effects in various degrees in different animal hosts. Referring especially to Mediterranean leishmaniasis this theory explains the acute forms, the chronic forms and the other spontaneously curable forms which have been distinguished in 
this disease. I think that in the Mediterranean regions leishmaniasis may be much more frequent than has yet been ascertained, and that slight cases, not easily to be diagnosed, may be particularly frequent.

The facts obtained by my long-continued researches into visceral leishmaniasis in the Mediterranean regions, the epidemiological factors of the close relations between leishmaniasis in children and in dogs, the close relations of contact between children and dogs affected with leishmaniasis and fleas infected with parasitic protozoa which are morphologically and biologically indistinguishable from Leishmania, afford ever-increasing confirmation of the results of my investigations, and tend to prove that the visceral leishmaniasis of the Mediterranean is produced by a species of Herpetomonas of Pulex irritans and Ctenocephalus canis which has adapted itself to live in children and dogs, themselves the habitual hosts of these fleas.

\section{BIBLIOGRAPHY.}

Alvares, D. (1911). Med. Contemp.

ArChibald, R. G. (1914). Journ. Roy. Army Med. Corps.

BaLFOUR (1906). Journ. Hygiene.

BaSILE, C. (1910-15). Rend. Acc. Lincei. Rome.

- (1913). Policlinico (Sezione Pratica).

(1912). Bull. Soc. Pathol. Exotique.

(1915). Annali d' Igiene.

Basile, C. and Visentini, A. (1912). Rend. Acc. Lincei. Rome.

Chagas, C. (1910, 1911). Mem. do Inst. Oswaldo Cruz.

Chatton (1919). Bull. Soc. Pathol. Exotique.

Fantham, H. B. (1912). Brit. Med. Assn. Liverpool.

- (1912). Brit. Med. Journ.

- (1913). Bull. Soc. Pathol. Exotique.

- (1915). Ann. Tropical Med. and Parasitol.

Fantham, H. B. and Porter, A. (1915). Proc. Cambridge Philos. Soc.

- (1915). Ann. Tropical Med. and Parasitol.

Lafont (1912). Ann. Institut Pasteur.

Laveran, A. and Francuini, G. (1913-14). Compt. Rend. Acad. Sci.

- $(1914,1915,1920)$. Bull. Soc. Pathol. Exotique.

Letshman, W. B. (1915). Journ. Roy. Army Med. Corps.

LÉGER (1918). C.R.S. B.

Mackinnon, A. (1909). Parasitology.

MarzocCht (1911). Pathologica.

Mrnchin, F. A. (1912). An Introduction to the Study of the Protozoa.

Minchin, E. A. and Thomson (1915). Quart. Journ. Microsc. Sci.

Nicolle, C. (1908-12). Arch. Inst. Pasteur de Tunis.

(1908). Compt. Rend. Acad.

- (1909). Ann. Inst. Pasteur.

NöfLuer W. (1912-14). Arch. f. Protistenk.

Patton, W. S. (1915). Text Book of Medical Entomology.

- (1908). Report of the Bacteriological Section, Royal Institute of Preventive Medicine. Madras.

(1908). Parasitology.

(1908). Arch.f. Protistenk. 
PARASITOLOGY, VOL. XII. NO. 4

PLATE XXI

$6^{1} 3^{2} \quad 0^{3}$

$3^{4}$

5

$\because$

Qi
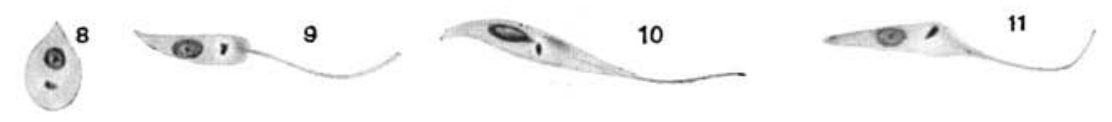

12

$00^{13}$

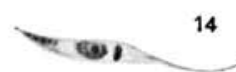

15

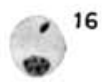

17

18

19

20

21

$22 ?$

$0^{23}$

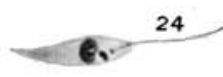

25

26

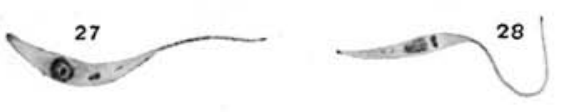

29

30

(c) ${ }^{31} \quad 3^{32}$

33

$6^{34}$
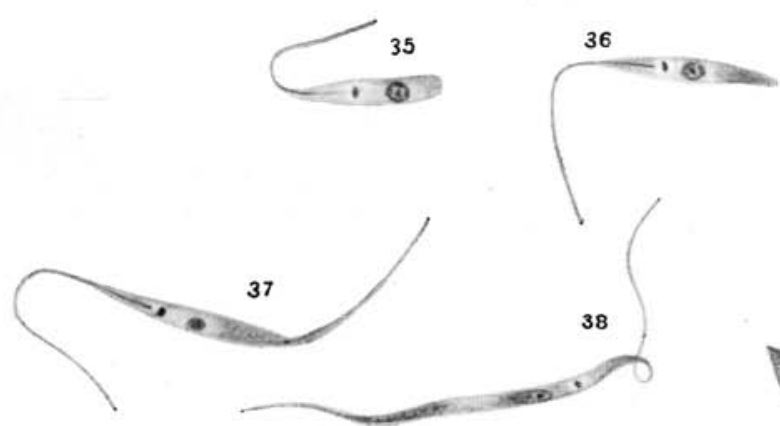

38

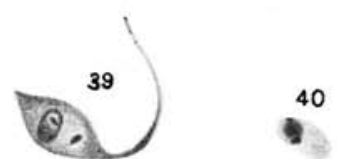

A. Mont ad nat. del. 
PARASITOLOGY, VOL. XII. NO. 4

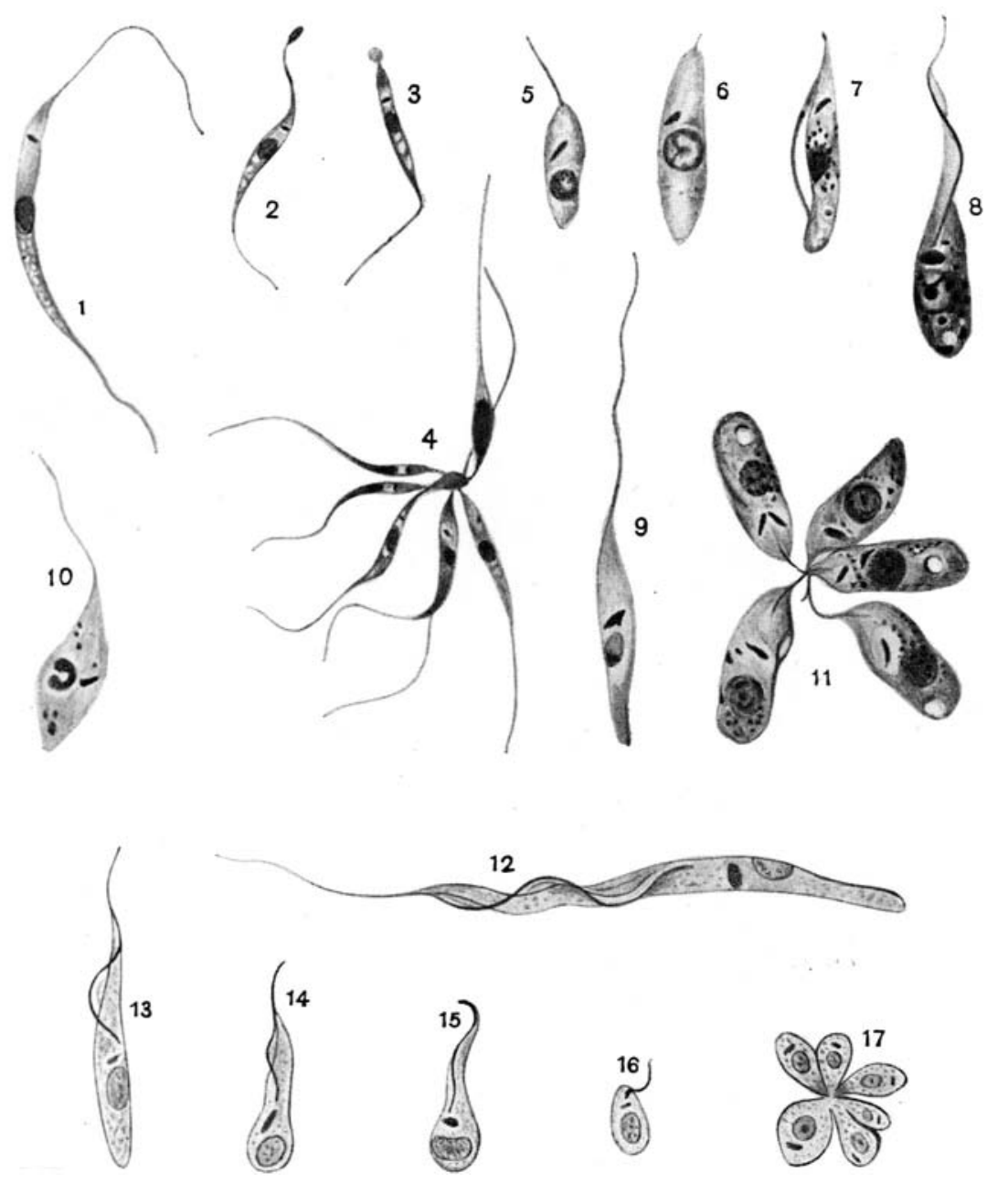




\section{BASILE}

PATtON, W. S.(1909). Lancet.

(1909). Trans. Soc. Tropical Med.

Patton, W. S. and Strickland (1908). Parasitology.

Porter, H. (1911). Parasitology.

RoGERs, L. (1904). Quart. Journ. Microsc. Sci.

Row, R. (1912). Brit. Med. Journ.

Sangiorar, G. (1911). Pathologica.

Sergent, E. (1912). Bull. Soc. Pathol. Exotique.

Ed. and Et. Sergent, A. Lhéritier and G. Lématre (1912). Bull. Soc. Pathoi. Exotique. Teiera, E. (1919). Bull. Soc. Pathol. Exotique.

Visentini, A. (1910). Studii intorno ad alcune malattie tropicali della Calabria e della Sicilia. Rome.

Wenyon, C. M. (1912-13). Journ. London School of Tropical Med.

- (1910). Parasitology III.

\section{EXPLANATION OF PLATES XXI AND XXII.}

\section{PLATE XXI.}

(a) Leishmania in the vertebrate host:

Figs. 1-4. Non-flagellate stage.

Figs. 5, 6. Showing homomorphous division.

Fig. 7. Showing heteromorphous division.

(b) Leishmania in cultures:

Fig. 8. Young non-Hagellate parasite.

Fig. 9. Flagellate form derived from non-flagellate parasites

Figs. 13, 14. Parasites commencing equal longitudinal division.

Fig. 15. Form derived from flagellate parasites by longitudinal division.

(c) Leishmania in Ctenocephalus canis:

Figs. 16-19. Pre-flagellate stages.

Figs. 20-22. Pre-flagellate stages showing equal longitudinal division.

Figs. 24-29. Flagellate stage.

Fig. 28. Parasite commencing equal longitudinal division.

Fig. 30. Parasite approaching the post-flagellate stage.

Figs. 31-34. Post-flagellate stage.

(d) Herpetomonas in Pulex irritans (a specimen kindly sent me by Dr Wenyon):

Figs. 35-39. Different stages of flagellate parasites in the flea's intestine.

Fig. 40. Parasite in post-flagellate stage.

\section{PLATE XXII.}

Figs. 1-7. Herpetomonas ctenophthalmi (after Mackinnon).

Figs. 8-11. Crithidia hystricopsillae (after Mackinnon).

Figs. 12-17. Crithidia pulicis (after Porter). 